\title{
Detection of a cryptic NUP214/ABL1 gene fusion by mate-pair sequencing (MPseq) in a newly diagnosed case of pediatric T-lymphoblastic leukemia
}

\author{
Jess F. Peterson, ${ }^{1}$ Beth A. Pitel, ${ }^{1}$ Stephanie A. Smoley, ${ }^{1}$ James B. Smadbeck, ${ }^{2}$ \\ Sarah H. Johnson, ${ }^{2}$ George Vasmatzis, ${ }^{2}$ Sarah J. Koon, ${ }^{1}$ Matthew R. Webley, ${ }^{1}$ \\ Mary McGrath, ${ }^{3}$ Michael G. Bayerl, ${ }^{4}$ Linda B. Baughn, ${ }^{1}$ Ross A. Rowsey, ${ }^{1}$ \\ Rhett P. Ketterling, ${ }^{1}$ Patricia T. Greipp, ${ }^{1}$ and Nicole L. Hoppman ${ }^{1}$

\footnotetext{
${ }^{1}$ Division of Laboratory Genetics and Genomics, Department of Laboratory Medicine and Pathology; ${ }^{2}$ Center for Individualized Medicine-Biomarker Discovery, Mayo Clinic, Rochester, Minnesota 55905, USA; ${ }^{3}$ Division of Pediatric Hematology/Oncology, Department of Pediatrics, Penn State Children's Hospital, Hershey, Pennsylvania 17033, USA; ${ }^{4}$ Department of Pathology and Laboratory Medicine, Penn State Milton
} \\ S. Hershey Medical Center, Hershey, Pennsylvania 17033, USA
}

Corresponding author: hoppman.nicole@mayo.edu

(C) 2019 Peterson et al. This article is distributed under the terms of the Creative Commons Attribution-NonCommercial License, which permits reuse and redistribution, except for commercial purposes, provided that the original author and source are credited.

Ontology term: T-cell acute lymphoblastic leukemias

Published by Cold Spring Harbor Laboratory Press

doi:10.1101/mcs.a003533
Abstract T-cell acute lymphoblastic leukemia (T-ALL) is an aggressive hematopoietic neoplasm involving the bone marrow and blood that accounts for $15 \%$ of childhood and $25 \%$ of adult ALL. Whereas multiple, recurrent genetic abnormalities have been described in T-ALL, their clinical significance is unclear or controversial. Importantly, $A B L 1$ rearrangements, most commonly described in BCR/ABL1-positive B-ALL and BCR-ABL1-like B-ALL, have been observed in T-ALL and may respond to tyrosine kinase inhibitor (TKI) therapy. We describe a newly diagnosed case of pediatric T-ALL with a fluorescence in situ hybridization abnormality suggesting a partial $A B L 1$ deletion by a $B C R / A B L 1$ dual-color dualfusion probe but that demonstrated a normal result using an $A B L 1$ break-apart probe. Mate-pair sequencing (MPseq), a next-generation sequencing (NGS)-based technology utilized to detect copy number and structural abnormalities with high resolution and precision throughout the genome, was performed and revealed a NUP214/ABL1 gene fusion that has been demonstrated to be sensitive to TKI therapy. This case demonstrates the power of MPseq to resolve chromosomal abnormalities unappreciable by traditional cytogenetic methodologies and highlights the clinical value of this novel NGS-based technology.

\section{INTRODUCTION}

T-cell acute lymphoblastic leukemia (T-ALL), a neoplasm of lymphoblasts committed to the T-cell lineage involving the bone marrow and blood, is observed in 15\% and $25 \%$ of childhood and adult ALL, respectively (You et al. 2015; Borowitz et al. 2017).

In general, childhood T-ALL is a clinically aggressive neoplasm that requires intensive chemotherapy regimens and is associated with a higher risk of induction failure and early relapse when compared to childhood B-ALL (Borowitz et al. 2017). Although recurrent genetic abnormalities in many hematologic neoplasms add diagnostic, prognostic, and therapyrelated value, the clinical significance of the known recurrent genetic abnormalities observed in T-ALL are mostly unclear or controversial (Borowitz et al. 2017; Taylor et al. 2017). 
COLD SPRING HARBOR Molecular Case Studies
NUP214/ABL1 gene fusion detection by MPseq
Interestingly, the detection of $A B L 1$ gene rearrangements, which are often associated with BCR/ABL1-positive B-ALL and BCR-ABL1-like (Ph-like) B-ALL (Pui et al. 2017; Tasian et al. 2017), have also been described in T-ALL_namely, NUP214/ABL1—and may be amenable to tyrosine kinase inhibitor (TKI) therapy (Graux et al. 2004; Burmeister et al. 2006; QuintásCardama et al. 2008; Deenik et al. 2009; Graux et al. 2009; Hagemeijer and Graux 2010; Clarke et al. 2011; De Braekeleer et al. 2011; Duployez et al. 2016; Simioni et al. 2016; Chen et al. 2017; Liu et al. 2017). Considering the limited treatment options and overall unfavorable prognosis of T-ALL, detecting genetic abnormalities that may respond to targeted therapy is critical.

Herein, we describe the detection of a cryptic NUP214/ABL1 gene fusion in a newly diagnosed case of pediatric T-ALL by mate-pair sequencing (MPseq), a unique next-generation sequencing (NGS)-based technology that can detect both chromosomal structural and copy number abnormalities with significantly increased resolution and precision when compared to conventional chromosome and fluorescence in situ hybridization (FISH) methodologies (Johnson et al. 2018; Smadbeck et al. 2018).

\section{RESULTS}

\section{Clinical Presentation}

The patient is a 4-yr-old male with a prior diagnosis of autism spectrum disorder who presented with a 2-wk history of extreme fatigue and significant bruising. On exam he had pallor, splenomegaly, and hepatomegaly, with the splenic tip palpated just below the umbilicus, and the hepatic border palpated at the costal edge. In addition, he had several subcentimeter cervical and inguinal lymph nodes. Petechiae were noted overlying the anterior upper legs and beneath both eyelids, and large bruises were noted over the legs bilaterally and over the midthoracic vertebrae. Initial laboratory results revealed leukocytosis, anemia, and thrombocytopenia. His presenting pertinent labs were as follows: white blood cell count $480 \mathrm{~K} / \mu \mathrm{L}$, hemoglobin $4.8 \mathrm{~g} / \mathrm{dL}$, platelets $23 \mathrm{~K} / \mu \mathrm{L}$, blasts $83 \%$, absolute blast count $399 \mathrm{~K} / \mu \mathrm{L}$, absolute neutrophil count $0 \mathrm{~K} / \mu \mathrm{L}$, lactate dehydrogenase $8574 \mathrm{U} / \mathrm{L}$, uric acid $6.6 \mathrm{mg} / \mathrm{dL}$, potassium $4.0 \mathrm{mEq} / \mathrm{L}$, phosphorus $4.2 \mathrm{mg} / \mathrm{dL}$, ionized calcium $1.45 \mathrm{mg} / \mathrm{dL}$, INR 1.7, and PT 20.3. A chest $X$ ray obtained at presentation revealed no mediastinal mass. The patient was admitted to the pediatric intensive care unit for management of anticipated tumor lysis syndrome given the significant leukocytosis.

Peripheral blood was sent for morphologic evaluation and flow cytometry studies. Normocytic normochromic anemia with mild anisopoikilocytosis was observed with absence of rouleaux and nucleated red blood cells. Marked leukocytosis with predominantly lymphoblasts and rare mature lymphocytes were observed with virtually absent neutrophils, monocytes, and platelets (Fig. 1). Flow cytometry of peripheral blood showed blasts represented 95\% of total leukocytes and expressed the following antigens: CD1a (partial dim), CD2, cCD3, double positive for CD4 and CD8, CD5, CD7, CD10, CD34 (partial dim), CD56, and TdT (Fig. 2). Blasts were negative for CD19, CD22, and MPO. Based on morphologic and flow cytometry results, a diagnosis of T-ALL was rendered. Cerebral spinal fluid was sampled and found to be negative for malignant cells, classifying the patient as CNS1. Of note, he did not receive corticosteroids within 4 wk prior to his diagnostic lumbar puncture. In addition, he did not have testicular disease appreciated on exam. A bone marrow biopsy and aspirate were obtained for morphologic assessment, chromosome studies, and FISH studies. The bone marrow aspirate and biopsy were hypercellular (95\%-100\%) and predominantly composed of lymphoblasts (90\%-95\% of marrow cellularity). Myeloid and erythroid lineages and megakaryocytes were all markedly decreased. 


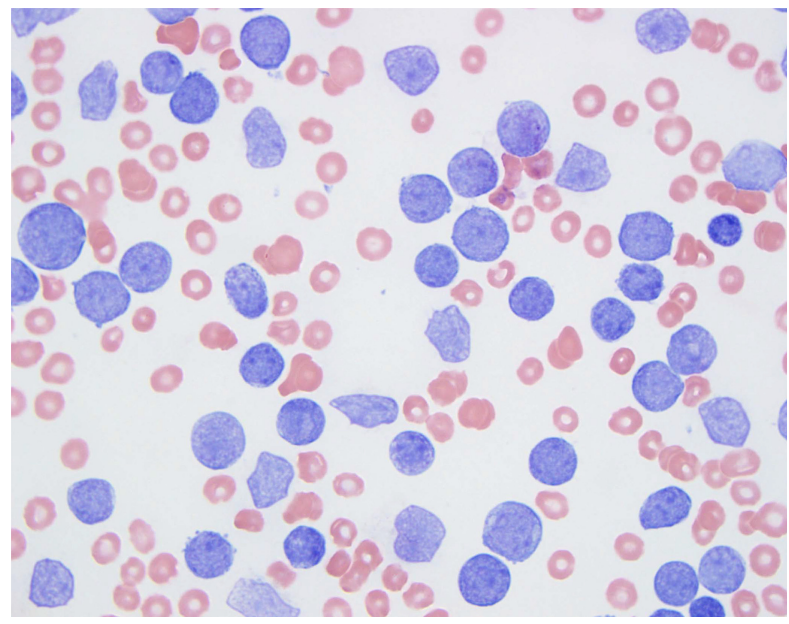

Figure 1. The peripheral blood smear showed marked leukocytosis with predominantly lymphoblasts and rare mature lymphocytes. Neutrophils, monocytes, and platelets were virtually absent.
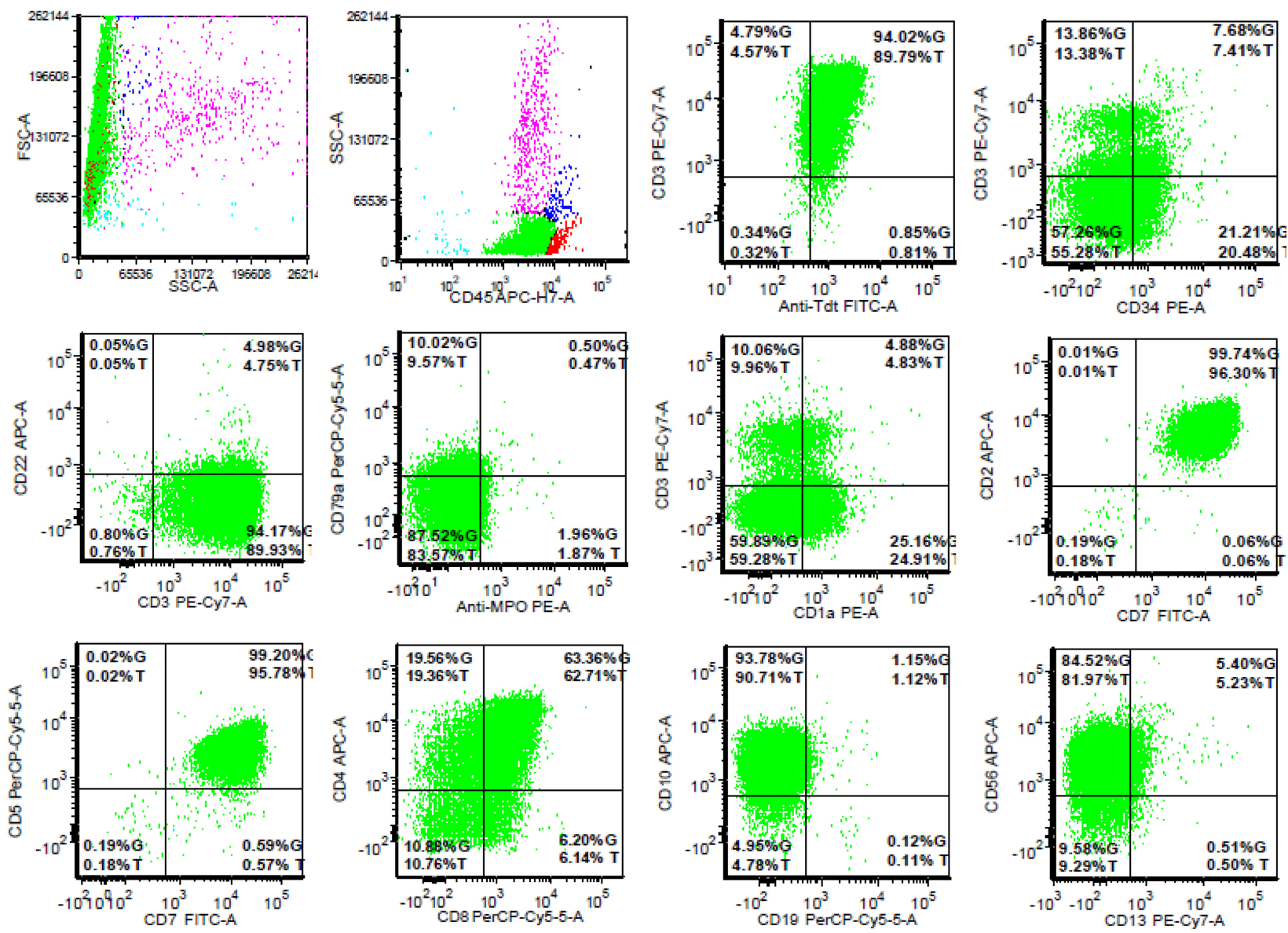

Figure 2. Pertinent immunophenotype results by flow cytometry indicating T-lymphoblastic leukemia. The lymphoblast population expressed the following antigens: CD1a (partial dim), CD2, cCD3, double positive for CD4 and CD8, CD5, CD7, CD10, CD34 (partial dim), CD56, and TdT. (Green) Blasts, (red) mature lymphocytes, (dark blue) monocytes, (magenta) granulocytes, (light blue) erythrocytes. 


\section{Genomic Analyses}

All genomic studies were performed on the submitted diagnostic bone marrow aspirate specimen. Conventional chromosome studies indicated 20 normal metaphases $(46, X Y)$ from an unstimulated culture (Fig. 3A), whereas T-ALL panel FISH studies revealed both complete and partial CDKN2A deletions in the majority of nuclei ( $96.5 \%$ of 200 interphase cells) (Fig. 3B) and what appeared to be a partial ABL1 deletion (89.4\% of 500 interphase cells; observed using a BCR/ABL1 D-FISH probe set) (Fig. 3C). Importantly, the remaining T-ALL and TKI responsive FISH probes were negative (see Methods for a complete list of FISH probes), including the ABL1 BAP probe set (Fig. 3D).

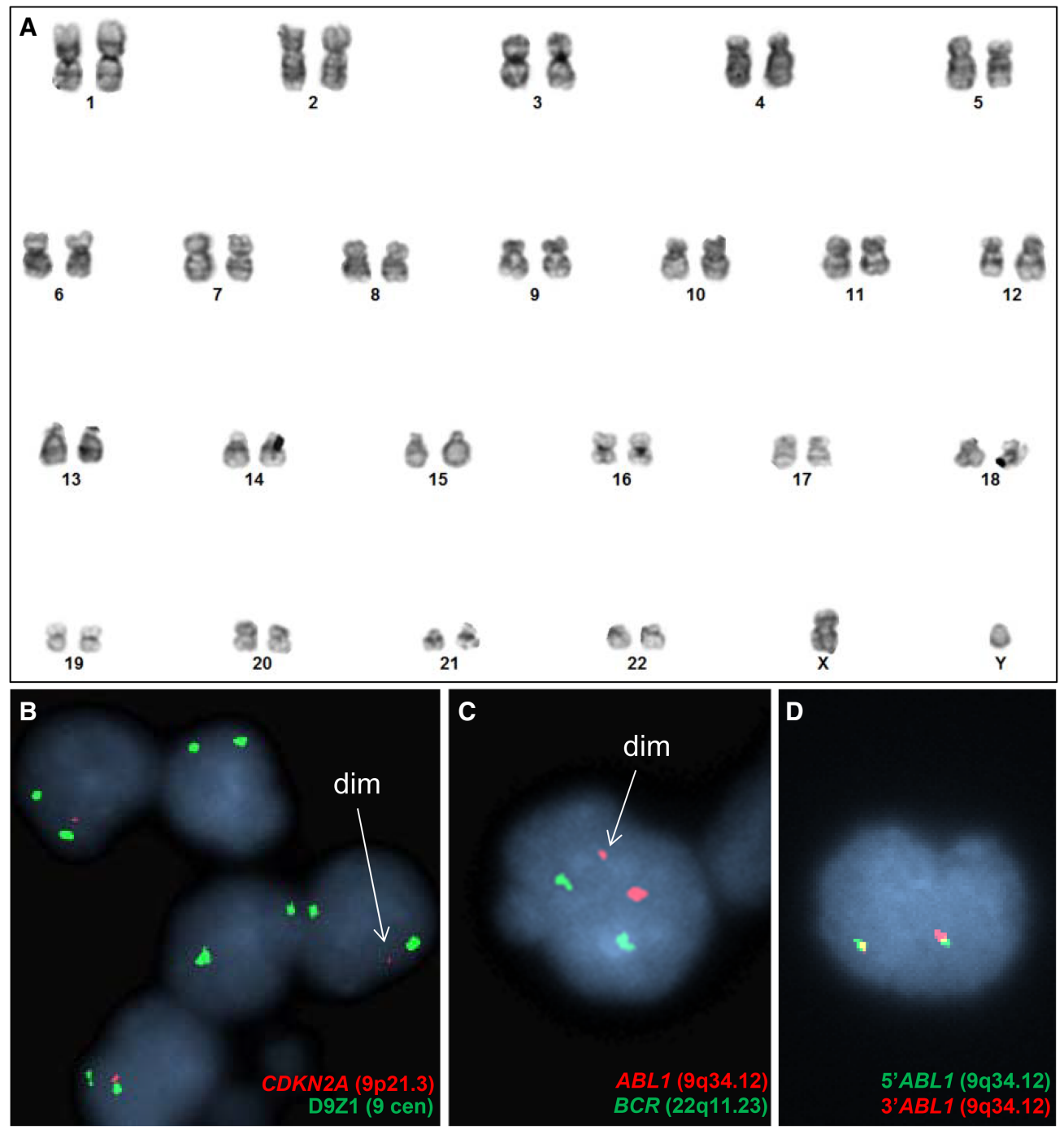

Figure 3. (A) Representative normal male karyogram $(46, \mathrm{XY})$ demonstrating no visible chromosome abnormalities. (B) Representative interphase cells showing either homozygous CDKN2A deletions (loss of both red signals) or a complete and partial loss of the CDKN2A gene regions (loss of a single red signal and a diminished red signal). (C) Representative interphase cell showing a diminished $A B L 1$ (red) signal using the $B C R / A B L 1 D-F I S H$ probe set (indicated by an arrow). This finding was initially interpreted as a partial deletion of the $A B L 1$ gene region. (D) Representative interphase cell showing two intact $A B L 1$ signals using the $A B L 1$ BAP FISH probe, interpreted as no disruption of the $A B L 1$ gene. 


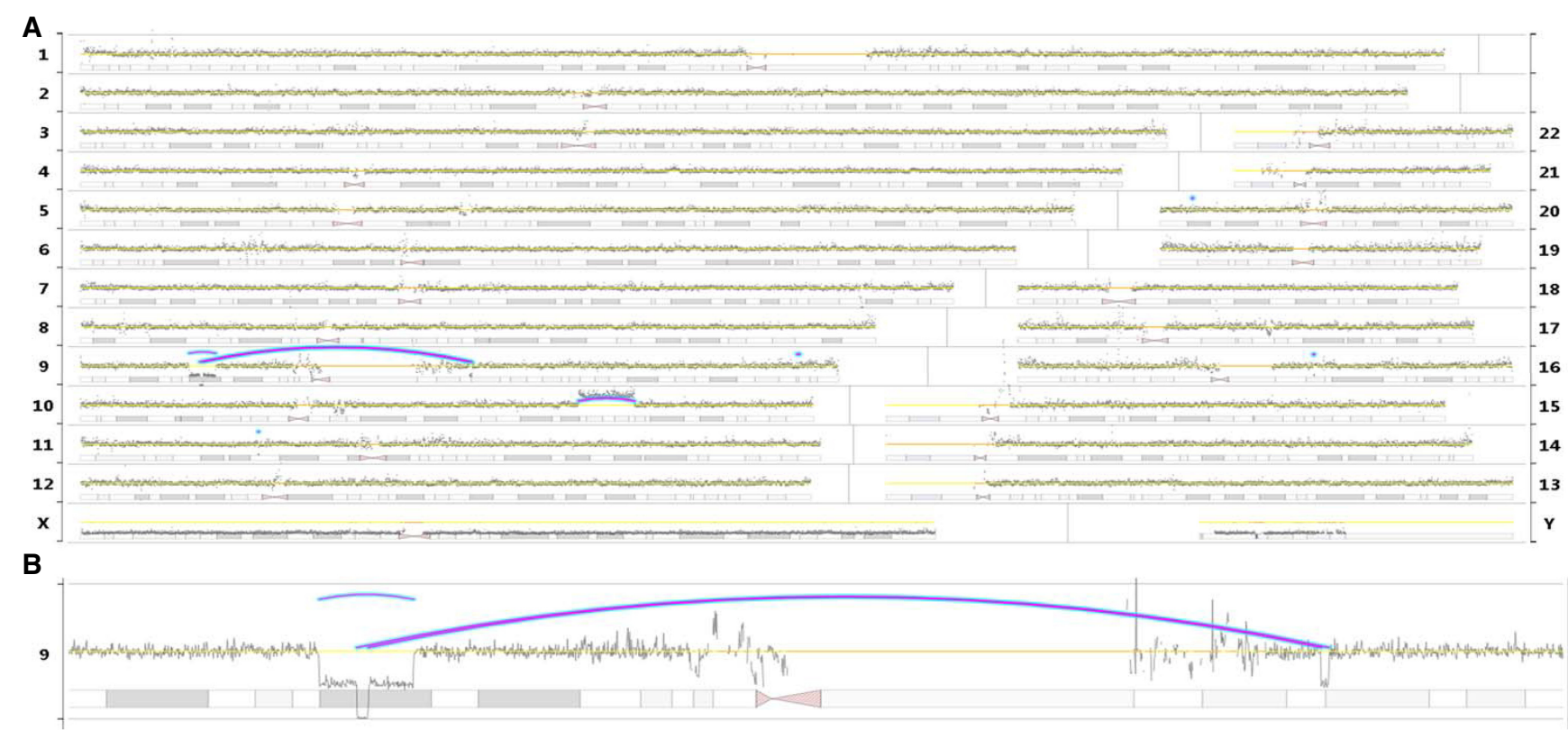

Figure 4. (A) Mate-pair sequencing (MPseq) genome plot demonstrating several structural and copy number abnormalities, including a pericentric inv(9), a heterozygous 9p21.3 deletion, and a 10.1-Mb gain of 10q. (B) Focused view of Chromosome 9 showing in greater detail the pericentric inv(9), including the deletions spanning the inversion breakpoints (9p21.3 and 9q21.12) and a 3.8-Mb deletion of the 9p21.3 chromosome region (including CDKN2A).

To further characterize the abnormal clone, MPseq was performed and revealed complex rearrangements involving both Chromosome 9 homologs and a 10.1-Mb duplication on 10q (Fig. 4A). A 3.8-Mb deletion of 9p (Chr 9:20,866,257-24,692,658) was observed on one Chromosome 9 homolog, resulting in loss of multiple genes including CDKN2A and CDKN2B (Fig. 4B). In addition, a pericentric inversion of the other Chromosome 9 homolog was observed that resulted in deletions at the breakpoint sites (9p21.3 and 9q21.12), including an 583-kb deletion (Chr 9:21,805,717-22,389,662) that also spanned CDKNA and CDKN2B (Fig. 4B). These results were consistent with homozygous CDKN2A deletions observed by FISH analysis. Lastly, a "cryptic" $t(9 ; 9)(q 34.13 ; q 34.12)$ was observed with breakpoints located in intron 31 of NUP214 (NM_001318324.1) and intron 1 of ABL1 (NM_007313.2) and is predicted to create an in-frame chimeric gene consisting of exons 1-31 of NUP214 and exons 2-11 of ABL1 (Fig. 5). Sanger sequencing confirmed the translocation breakpoints indicated by MPseq (Table 1).

\section{Treatment Outcomes}

The patient was initially treated with methylprednisolone for $72 \mathrm{~h}$ for gentle leukoreduction followed by systemic chemotherapy as per the Children's Oncology Group (COG) AALL1231 trial protocol. As the family declined to enroll in the trial, the patient was treated off study as per the standard therapy arm. Induction chemotherapy consisted of the standard four-drug regimen of vincristine, dexamethasone, daunorubicin, and pegaspargase, and the patient also received standard CNS prophylaxis with intrathecal cytarabine and methotrexate. Peripheral blasts were not cleared from circulation until day 14 of induction therapy. During induction, he experienced multiple complications, including steroid-induced hypertension requiring several antihypertensives, vincristine-induced syndrome of inappropriate antidiuretic hormone secretion, sepsis due to methicillin-sensitive Staphylococcus aureus, 


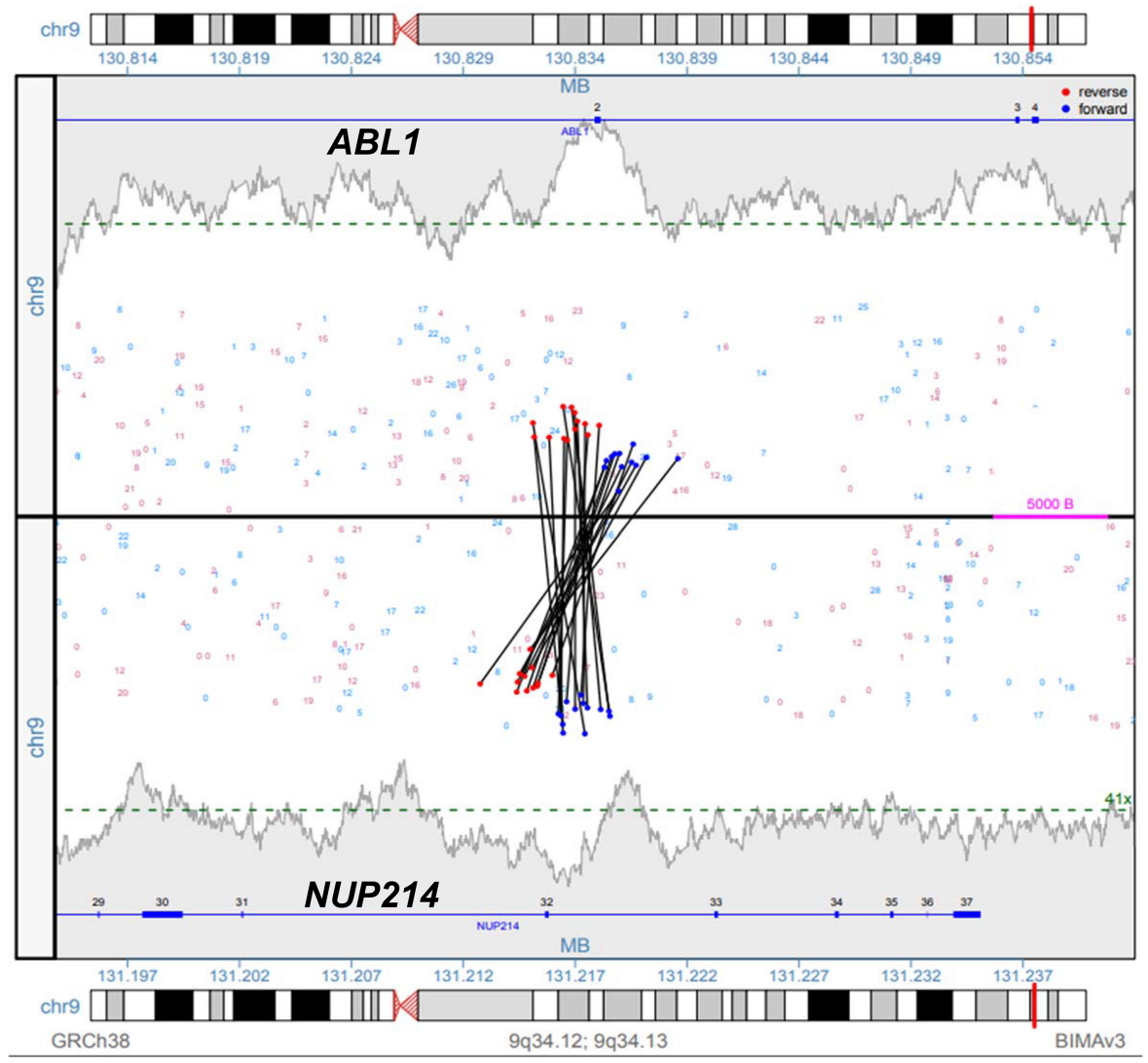

Figure 5. Mate-pair sequencing (MPseq) results visualized in SVAtools. Junction plot demonstrating a translocation between the NUP214 gene (intron 31; NM_001318324.1) at 9q34.13 and the ABL1 gene (intron 1; NM_007313.2) at 9q34.12. This translocation is predicted to create an in-frame chimeric gene consisting of exons 1-31 of NUP214 and exons 2-11 of ABL1.

and a large saddle embolus. Despite these complications, therapy was not withheld. At the end of induction, a bone marrow study revealed measurable minimal residual disease (MRD) of $0.006 \%$. Per the AALL1231 protocol, patients with who MRD $<0.01 \%$ at the end of induction that are CNS1 with no testicular involvement and who did not receive steroid pretreatment prior to the diagnostic lumbar puncture are stratified into the standard risk group. Consolidation therapy consisted of cyclophosphamide, cytarabine, mercaptopurine, vincristine, and pegaspargase. The patient tolerated consolidation therapy well and achieved remission as evidenced by an end of consolidation MRD of $0 \%$. Since obtaining remission,

Table 1. Mate-pair sequencing (MPseq) and Sanger sequencing results for the NUP214/ABL1 gene fusion

\begin{tabular}{|c|c|c|c|c|c|c|c|c|c|}
\hline Gene 1 & Gene 2 & Position 1 & Position 2 & Transcript 1 & $\begin{array}{l}\text { Intron } \\
\text { number } \\
1\end{array}$ & Transcript 2 & $\begin{array}{l}\text { Intron } \\
\text { number } \\
2\end{array}$ & Fusion junction sequence ${ }^{a}$ & $\begin{array}{l}\text { Frame- } \\
\text { shift } \\
\text { class }\end{array}$ \\
\hline$A B L 1$ & NUP214 & Chr 9:130,835,718 & Chr 9:131,215,806 & NM_007313.2 & 1 & NM_001318324.1 & 31 & $\begin{array}{l}\text { CAGTTCTCTTATATTCTGTCTCTC } \\
\text { TTTCTTTCTCTCTGTGcgaatttttt } \\
\text { CCCAAAAGTTGAAGCCTTT } \\
\text { AATATTTCTCACCTCTTACAT }\end{array}$ & In-frame \\
\hline
\end{tabular}

${ }^{a}$ Bold, uppercase sequence maps to gene 1. Sequence in lowercase is found at the breakpoint but does not map to either chromosome. Nonbolded uppercase sequence maps to gene 2 . 
this patient has completed the interim maintenance and delayed intensification phases of therapy as per the nonrandomized, standard risk arm of the COG AALL1231 protocol. The patient is currently in his second phase of maintenance therapy and is doing well clinically with no signs of relapse.

\section{DISCUSSION}

Following conventional chromosome and T-ALL and TKI panel FISH studies that yielded little prognostic and treatment-related information, MPseq was performed to further elucidate what appeared to represent a partial $A B L 1$ deletion by FISH analysis using the $B C R /$ $A B L 1$ D-FISH probe set. Rather than confirming a partial or complete deletion as suggested by the ABL1 D-FISH probe footprint, a NUP214/ABL1 gene fusion resulting from a "cryptic" $\mathrm{t}(9 ; 9)(q 34.13 ; q 34.12)$ was revealed by MPseq and subsequently confirmed by Sanger sequencing. This discrepant result was resolved after the translocation breakpoints by MPseq were compared to the ABL1 D-FISH footprint. Although a segment of the disrupted ABL1 D-FISH probe on der(9) resulted in a diminished (dim) $A B L 1$ signal, the intact $A B L 1$ D-FISH probe on der(9) was enhanced via juxtaposition of the translocated portion of the ABL1 D-FISH probe from the der(9) (Fig. 6).

Similarly, because the ABL1 BAP generated two apparently normal, intact fusion signals, the ABL1 BAP FISH footprints were compared to the NUP214/ABL1 breakpoints. Although the $A B L 1$ gene on the der(9) was disrupted because of the translocation event and should be associated with a disruption of the ABL1 BAP, the MPseq results indicate a fusion signal was ultimately "re-created" on the der(9) because of retention of the 5'ABL1 BAP from the der(9) with juxtaposition of the translocated portion of the 3'ABL1 BAP from the der(9), thus "masking" the ABL1 rearrangement (Fig. 6). The initial break on the der(9) disrupts the NUP214 gene rather than the $A B L 1$ gene and is not associated with a true disruption of the BAP $A B L 1$ probe (Fig. 6). However, the der(9) is ultimately the location of the NUP214/ABL1 fusion that is generated by the "cryptic" $t(9 ; 9)$ event. Importantly, amplification of the NUP214/ $A B L 1$ fusion gene appears to be required for oncogenicity and may be a late event occurring in only a subpopulation of cells (Graux et al. 2009). Although amplification of the NUP214/ $A B L 1$ fusion gene was not observed in our case, follow-up testing to detect clonal evolution would be of clinical importance.

In addition to the NUP214/ABL1 rearrangement, MPseq identified heterozygous and homozygous $9 p$ deletions, a pericentric inv(9) and a partial gain of 10q. Taken together, of the multiple structural and numerical abnormalities identified by MPseq, FISH only identified complete and partial CDKN2A deletions, whereas chromosome studies were normal.

MPseq is a unique NGS-based technology that provides significantly higher resolution and precision when compared to chromosome and FISH methodologies, often characterizing breakpoints to the exact intron or exon. Library preparation involves circularizing long DNA fragments ( $\sim 2-5 \mathrm{~kb})$, followed by paired end sequencing of the mate-pair fragments. Rather than direct sequencing, long insert sequences are inferred from short paired end reads, thus allowing for the robust detection of structural abnormalities without extensive sequencing. Importantly, MPseq leverages inferred sequence in between reads to achieve "bridged coverage"; thus the amount of sequencing necessary to gather evidence for structural abnormalities and the resulting cost are less compared to traditional paired end sequencing across the entire genome.

Utilization of bridge coverage also allows for a breakpoint resolution smaller than fragment size, typically within a 1-kb window (or smaller, depending on the coverage of the study). However, MPseq also has the potential to capture "split reads," wherein a single sequence read, rather than a set of paired reads, contains sequences from both sides of 
COLD SPRING HARBOR Molecular Case Studies
NUP214/ABL1 gene fusion detection by MPseq
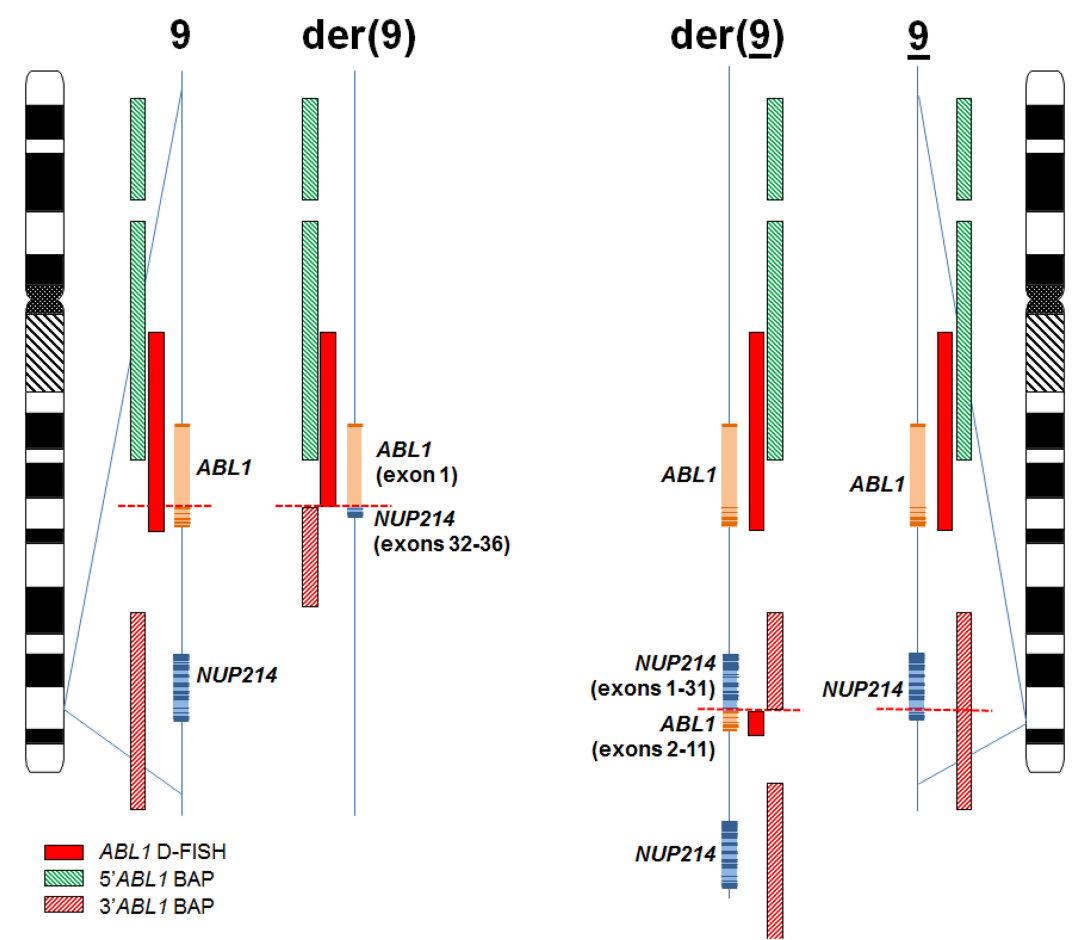

Figure 6. A focused view of the ABL1 and NUP214 gene regions on the derivative copies of Chromosomes 9. Horizontal dashed red lines indicate the breakpoints on each derivative Chromosome 9 and disruption of each FISH probe footprint. The solid red vertical bars indicate the ABL1 FISH probe footprint from the $B C R / A B L 1$ D-FISH probe set (ABL1 D-FISH). The close proximity of ABL1 and NUP214 on each Chromosome 9 resulted in a diminished $A B L 1$ signal on the der(9) homolog while enhancing the $A B L 1$ FISH probe signal intensity on the der(9) homolog. The striped vertical green bars indicate the $5^{\prime} A B L 1 \mathrm{FISH}$ probe footprint from the $A B L 1 \mathrm{BAP}\left(5^{\prime} A B L 1 \mathrm{BAP}\right)$ and the striped vertical red bars indicate the $3^{\prime} A B L 1 \mathrm{FISH}$ probe footprint from the ABL1 BAP ( $\left.3^{\prime} A B L 1 \mathrm{BAP}\right)$. Although the ABL1 gene was rearranged on the der(9) homolog, the $3^{\prime} A B L 1$ BAP spanning the NUP214 rearrangement from the der(9) homolog created a fusion signal on the der(9), thus "masking" the ABL1 rearrangement.

the rearrangement. These split reads identify the breakpoint down to the base pair level, essentially providing the maximum level of resolution without requiring follow-up Sanger sequencing. The ability to detect both structural and copy number abnormalities with high resolution and precision significantly exceeds the detection capabilities of $\mathrm{FISH}$, as we have demonstrated in this case. Last, the ability to detect structural rearrangements of clinical significance will continue to grow as disease-specific and potentially targetable abnormalities are discovered. For example, the list of kinase genes that respond to TKI therapy in Ph-like B-ALL continues to grow (Pui et al. 2017; Tasian et al. 2017), and it will become infeasible or cost-effective to continue the development of FISH probes or other methodologies targeting single-gene regions for application to the rapidly growing list of gene rearrangements.

In summary, we have demonstrated the clinical utility of MPseq in a newly diagnosed case of pediatric T-ALL with a cryptic NUP214/ABL1 fusion. The detection of the NUP214/ $A B L 1$ gene fusion by MPseq, in addition to other structural and copy number abnormalities, were undetected by chromosome studies and two standard, clinical FISH probe sets with $A B L 1$ coverage, thus illustrating the power of this novel NGS-based technology. Although this patient has not yet been treated with TKI therapy, the ability of MPseq to reveal a genetic abnormality of potential clinical significance that was missed by standard cytogenetic 
COLD SPRING HARBOR Molecular Case Studies
NUP214/ABL1 gene fusion detection by MPseq evaluation has been demonstrated, and the NUP214/ABL1 fusion could provide an alternative treatment target if this patient experiences a T-ALL relapse.

\section{METHODS}

\section{Conventional Chromosome Analysis}

Cells from the bone marrow aspirate specimen were cultured, harvested, and banded utilizing standard cytogenetic techniques according to specimen-specific protocols. Twenty metaphases were analyzed by two qualified clinical cytogenetic technologists and interpreted by a board-certified (American Board of Medical Genetics and Genomics [ABMGG]) clinical cytogeneticist.

\section{Fluorescence In Situ Hybridization (FISH)}

The T-ALL and TKI-responsive FISH panels were performed on interphase nuclei including locus-specific (CDKN2A/D9Z1 [Abbott Molecular], TP53/D17Z1 [Abbott Molecular]), break-apart (KMT2A(MLL) [Abbott Molecular], TRB [laboratory-developed test, LDT], TRD [LDT], TAL1/STIL [LDT], ABL1 [LDT], PDGFR $\beta$ [LDT], JAK2 [LDT], ETV6 [LDT], NUP98 [LDT]), and D-FISH (BCR/ABL1 to detect ABL1 amplification [Abbott Molecular], TLX3/ $B C L 11 B$ [LDT], MLLT10/PICALM [LDT]) probe sets. The bone marrow aspirate specimen was subjected to standard FISH pretreatment, hybridization, and fluorescence microscopy according to specimen-specific protocols. FISH analysis was performed by two qualified clinical cytogenetic technologists and interpreted by a board-certified (ABMGG) clinical cytogeneticist.

\section{Mate-Pair Sequencing (MPseq)}

DNA was extracted from a fixed cell pellet and $1 \mu \mathrm{g}$ of DNA was utilized for mate-pair sequencing library preparation and was processed using the Illumina Nextera Mate Pair library kit (Illumina). Library preparation consisted of tagmentation to simultaneously shear and biotinylate the genomic DNA, strand displacement to fill any gaps left by the tagmentation step, and overnight circularization (16-20 h) to produce stabile 2- to 5-kb DNA fragments. AMPure purification (Beckman Coulter) was performed after the tagmentation and strand displacement steps $(0.56 \times$ and $0.4 \times$, respectively) to ensure only the longest fragments are selected to complete library preparation. After overnight circularization, noncircularized DNA was digested with exonuclease prior to mechanical shearing of the circularized fragments with a Covaris LE220 System (Covaris). The resulting biotinylated DNA fragments were bound to Dynabeads M-280 Streptavidin (Thermo Fisher Scientific) and subsequently processed through end repair, A-tailing, ligation of 7-bp Illumina adapters (a component of the TruSeq DNA library prep kit), and PCR using the PCR Primer Cocktail (Illumina) and KAPA HiFi HotStart Ready Mix PCR Kit (KAPA Biosystems). A 0.67× AMPure purification was performed to complete library preparation. MPseq libraries were multiplexed at two samples per lane to be sequenced on the Illumina HiSeq 2500 in rapid run mode. On both ends of each mate-pair fragment, 101 base pairs were sequenced to a bridged coverage of $43 \times$ and a base coverage of approximately $6 \times$. Data were aligned to the reference genome (GRCh38) using BIMAv3, and abnormalities were identified and visualized using SVAtools, an in-house developed bioinformatics tool. Additional information regarding MPseq technology and bioinformatics tools have been previously described (Drucker et al. 2014; Johnson et al. 2018; Smadbeck et al. 2018). 
COLD SPRING HARBOR Molecular Case Studies
NUP214/ABL1 gene fusion detection by MPseq
Competing Interest Statement

The authors have declared no competing interest.

Received September 26, 2018; accepted in revised form November 5, 2018.

\section{Sanger Sequencing}

Reference DNA sequences spanning the minimal 5' and maximal 3' positions of MPseq approximate breakpoints were used for primer design using Primer3Plus. End-point PCR was performed on patient DNA with a 50\% 2× Paq5000 Hotstart PCR Master Mix (Agilent) using touchdown PCR. Results were visualized on a $2 \%$ agarose gel in a UV light box, and amplicon sizes were estimated. Selected amplicons were purified using Exo-SAP-IT PCR Product Cleanup Reagent (Thermo Fisher Scientific) and Sanger sequencing was performed on a 3730x/ DNA Analyzer (Thermo Fisher Scientific). The resulting sequences were analyzed using Sequencher DNA Sequence Analysis Software (Gene Codes Corporation) and mapped to the GRCh38 genome using the BLAT function in the UCSC genome browser to determine precise breakpoints in this rearrangement.

\section{ADDITIONAL INFORMATION}

\section{Data Deposition and Access}

This variant was submitted to ClinVar (http://www.ncbi.nlm.nih.gov/clinvar) and can be found under accession number SCV000882548.

\section{Ethics Statement}

The results described in this manuscript were derived from clinical testing rather than research testing. Therefore, consent was not required for testing, and because patient identifiers were removed for the purposes of this manuscript, consent (oral or written) was not obtained. Mayo Clinic does not require IRB approval for the publication of single case reports.

\section{Author Contributions}

J.F.P. drafted and edited the manuscript. B.A.P., S.A.S., S.J.K., M.R.W., R.P.K., P.T.G., and N.L.H. analyzed the data and reviewed and edited the manuscript. J.B.S., S.H.J., G.V., and L.B.B. reviewed and edited the manuscript. M.M. and M.G.B. collected clinical and pathologic data and reviewed and edited the manuscript. R.A.R. participated in manuscript writing and reviewed and edited the manuscript.

\section{Funding}

Funding for this project was provided via internal Mayo Clinic funds; no external/National Institutes of Health funding was used.

\section{REFERENCES}

Borowitz MJ, Chan JKC, Downing JR, Le Beau MM. 2017. B-lymphoblastic leukaemia/lymphoma. In WHO classification of tumours of haematopoietic and lymphoid tissues (ed. Swerdlow SH, et al.), revised 4th ed., pp. 203-209. IARC, Lyon.

Burmeister T, Gökbuget N, Reinhardt R, Rieder H, Hoelzer D, Schwartz S. 2006. NUP214-ABL1 in adult T-ALL: the GMALL study group experience. Blood 108: 3556-3559. doi:10.1182/blood-2006-04-014514

Chen Y, Zhang L, Huang J, Hong X, Zhao J, Wang Z, Zhang K. 2017. Dasatinib and chemotherapy in a patient with early T-cell precursor acute lymphoblastic leukemia and NUP214-ABL1 fusion: a case report. Exp Ther Med 14: 3979-3984. doi:10.3892/etm.2017.5046

Clarke S, O'Reilly J, Romeo G, Cooney J. 2011. NUP214-ABL1 positive T-cell acute lymphoblastic leukemia patient shows an initial favorable response to imatinib therapy post relapse. Leuk Res 35: e131-e133. doi:10.1016/j.leukres.2011.03.025

De Braekeleer E, Douet-Guilbert N, Rowe D, Bown N, Morel F, Berthou C, Férec C, De Braekeleer M. 2011. $A B L 1$ fusion genes in hematological malignancies: a review. Eur J Haematol 86: 361-371. doi:10.1111/j .1600-0609.2011.01586.x 
Deenik W, Beverloo HB, van der Poel-van de Luytgaarde SC, Wattel MM, van Esser JWJ, Valk PJ, Cornelissen JJ. 2009. Rapid complete cytogenetic remission after upfront dasatinib monotherapy in a patient with a NUP214-ABL1-positive T-cell acute lymphoblastic leukemia. Leukemia 23: 627-629. doi:10.1038/leu 2008.318

Drucker TM, Johnson SH, Murphy SJ, Cradic KW, Thereau TM, Vasmatzis G. 2014. BIMA V3: an aligner customized for mate pair library sequencing. Bioinformatics 30: 1627-1629. doi:10.1093/bioinformatics/ btu078

Duployez N, Grzych G, Ducourneau B, Fuentes MA, Grardel N, Boyer T, Chahla WA, Bruno B, Nelken B, Clappier $E$, et al. 2016. NUP214-ABL1 fusion defines a rare subtype of B-cell precursor acute lymphoblastic leukemia that could benefit from tyrosine kinase inhibitors. Haematologica 101: e133-e134. doi:10.3324/ haematol.2015.136499

Graux C, Cools J, Melotte C, Quentmeier H, Ferrando A, Levine R, Vermeesch JR, Stul M, Dutta B, Boeckx N, et al. 2004. Fusion of NUP214 to ABL1 on amplified episomes in T-cell acute lymphoblastic leukemia. Nat Genet 36: 1084-1089. doi:10.1038/ng1425

Graux C, Stevens-Kroef M, Lafage M, Dastugue N, Harrison CJ, Mugneret F, Bahloula K, Struski S, Grégoire MJ, Nadal N, et al. 2009. Heterogeneous patterns of amplification of the NUP214-ABL1 fusion gene in T-cell acute lymphoblastic leukemia. Leukemia 23: 125-133. doi:10.1038/leu.2008.278

Hagemeijer A, Graux C. 2010. ABL1 rearrangements in T-cell acute lymphoblastic leukemia. Genes Chromosomes Cancer 49: 299-308. doi:10.1002/gcc.20743

Johnson SH, Smadbeck JB, Smoley SA, Gaitatzes A, Murphy SJ, Harris FR, Drucker TM, Zenka RM, Pitel BA, Rowsey RA, et al. 2018. SVAtools for junction detection of genome-wide chromosomal rearrangements by mate-pair sequencing (MPseq). Cancer Genet 221: 1-18. doi:10.1016/j.cancergen.2017.11.009

Liu Y, Easton J, Shao Y, Maciaszek J, Wang Z, Wilkinson MR, McCastlain K, Edmonson M, Pounds SB, Shi L, et al. 2017. The genomic landscape of pediatric and young adult T-lineage acute lymphoblastic leukemia. Nat Genet 49: 1211-1218. doi:10.1038/ng.3909

Pui CH, Roberts KG, Yang JJ, Mullighan CG. 2017. Philadelphia chromosome-like acute lymphoblastic leukemia. Clin Lymphoma Myeloma Leuk 17: 464-470. doi:10.1016/j.clml.2017.03.299

Quintás-Cardama A, Tong W, Manshouri T, Vega F, Lennon PA, Cools J, Gilliland DG, Lee F, Cortes J, Kantarjian $\mathrm{H}$, et al. 2008. Activity of tyrosine kinase inhibitors against human NUP214-ABL1-positive T cell malignancies. Leukemia 22: 1117-1124. doi:10.1038/leu.2008.80

Simioni C, Ultimo S, Martelli AM, Zauli G, Milani D, McCubrey JA, Capitani S, Neri LM. 2016. Synergistic effects of selective inhibitors targeting the PI3 K/AKT/mTOR pathway or NUP214-ABL1 fusion protein in human acute lymphoblastic leukemia. Oncotarget 7: 79842-79853. doi:10.18632/oncotarget.13035

Smadbeck JB, Johnson SH, Smoley SA, Gaitatzes A, Drucker TM, Zenka RM, Kosari F, Murphy SJ, Hoppman N, Aypar U, et al. 2018. Copy number variant analysis using genome-wide mate-pair sequencing. Genes Chromosomes Cancer 57: 459-470. doi:10.1002/gcc.5

Tasian SK, Loh ML, Hunger SP. 2017. Philadelphia chromosome-like acute lymphoblastic leukemia. Blood 130: 2064-2072. doi:10.1182/blood-2017-06-743252

Taylor J, Xiao W, Abdel-Wahab O. 2017. Diagnosis and classification of hematologic malignancies on the basis of genetics. Blood 130: 410-423. doi:10.1182/blood-2017-02-734541

You MJ, Medeiros LJ, Hsi ED. 2015. T-lymphoblastic leukemia/lymphoma. Am J Clin Pathol 144: 411-422. doi:10.1309/AJCPMF03LVSBLHPJ 


\title{
COLD SPRING HARBOR Molecular Case Studies
}

\section{Detection of a cryptic NUP214/ABL1 gene fusion by mate-pair sequencing (MPseq) in a newly diagnosed case of pediatric T-lymphoblastic leukemia}

\author{
Jess F. Peterson, Beth A. Pitel, Stephanie A. Smoley, et al.
}

Cold Spring Harb Mol Case Stud 2019, 5: a003533

Access the most recent version at doi: $10.1101 / \mathrm{mcs} . a 003533$

\begin{abstract}
References This article cites 19 articles, 4 of which can be accessed free at: http://molecularcasestudies.cshlp.org/content/5/2/a003533.full.html\#ref-list-1

License This article is distributed under the terms of the Creative Commons Attribution-NonCommercial License, which permits reuse and redistribution, except for commercial purposes, provided that the original author and source are credited.

Email Alerting Receive free email alerts when new articles cite this article - sign up in the box at the Service top right corner of the article or click here.
\end{abstract}

